\title{
Gattungstheorie als universelles Erklärungsmodell
}

Michail Bachtin: Sprechgattungen. Hgg. Rainer Grübel, Renate Lachmann, Sylvia Sasse. Übersetzt von Rainer Grübel und Alfred Sproede. Berlin: Matthes \& Seitz 2017 (=Batterien NF, 28), 336 S.

Unter dem Titel Sprechgattungen hat der Berliner Verlag eine aus Mitteln der Universität Zürich unterstützte Sammlung von Texten Michail M. Bachtins veröffentlicht, die erstens unterschiedliche Grade der Abgeschlossenheit aufweisen, zweitens in einem thematischen und drittens in einem chronologischen Zusammenhang stehen. Vieles davon liegt erstmals in deutscher Sprache vor; der dem Band den Titel gebende Aufsatz von 1953 in einer Neuübersetzung. Der Band beinhaltet:

- $\quad$ als Haupttext den Aufsatz Sprechgattungen (1953), S. 7-59;

- $\quad$ ergänzend dazu Übersetzungen der edierten Fassungen von Bachtins Vorarbeiten unter dem Titel Aus den Archiv-Niederschriften zur Arbeit "Sprechgattungen «; das sind ursprünglich in Hefte eingetragene Notizen aus den Jahren 1952-53, tw. fragmentarisch, tw. ausformuliert, jedenfalls nicht von Bachtin zur Veröffentlichung vorbereitet: Der Dialog S. 61-63, Dialog I. Die dialogische Rede S. 64-75, Dialog II S. 76-99, Vorbereitende Materialien S. 99-150; diese Texte folgen der russischen Ausgabe von Bachtins Gesammelten Werken (Bd. 5, Moskau 1996);

- $\quad$ mit Die Sprache in der künstlerischen Literatur eine weitere Skizze Bachtins (1954), die thematisch ähnliche Bereiche berührt, S. 151-164;

- $\quad$ sowie das Transkript eines Hefts mit Notizen, betitelt Sprache und Rede (1957-58), erstveröffentlicht 1991 und nicht in die genannte russische Werkausgabe aufgenommen, ${ }^{1}$ S. $165-172$;

- $\quad$ ein Nachwort der Herausgeberinnen, das unter dem Titel Dialogische Obertöne (S. 173-207) den Anhang eröffnet; dieser macht insgesamt 
in etwa die Hälfte des Bandes aus und schließt einen seitenstarken Anmerkungsteil ein (S. 209-325).

Diesem Konvolut ganz unterschiedlicher Texte, die zwar von einer Hand und aus einer abgegrenzten \Schaffensperiode`stammen, aber ganz unterschiedlichen Status zwischen Notiz, Entwurf, Skizze und Werk haben, ${ }^{2}$ lesend gerecht zu werden, ist keine triviale Aufgabe, vor allem wenn man auch noch die Untiefen der Übersetzung mit in Betracht ziehen muss: Sie muss ja nicht zuletzt auf bisherige Übersetzungen von Bachtins Texten Rücksicht nehmen, um Mehrdeutigkeiten und terminologischen Schwierigkeiten nach Möglichkeit vorzubauen, was gerade dort problematisch ist, wo der Autortext selbst Unschärfen in der Begriffsverwendung (etwa zwischen den Vorstufen und dem von Bachtin zur Veröffentlichung vorbereiteten Text Sprechgattungen) in Kauf nimmt bzw. es Teil von Bachtins eigener >dialogischer`Gedankenarbeit ist, an den Notaten Begriffe zu schärfen und sie zugleich zu überschreiben. All das nötigt dazu, die vorliegende Ausgabe zwar so ernst wie möglich zu nehmen, zugleich aber so vorsichtig wie nötig. Der Leser ist gezwungen, in ein ganzes - faszinierendes - Gedankengebäude einzusteigen, das im vorliegenden Band allerdings gegen die Entstehungslogik dargestellt ist (Empfehlung: Lektüre bei den »ArchivNiederschriften« beginnen).

Abseits editorischer Herausforderungen der übersetzenden BachtinPhilologie bietet der Band allerdings auch sechs gute Jahrzehnte nach der Entstehung des Materials ausreichend Stoff für eine ganze Reihe von Disziplinen. Bachtin, der schwer disziplinär einzuordnende Denker, wendet sich teilweise explizit gegen die zeitgenössische sowjetische Linguistik, aber auch gegen jene der Genfer Schule rund um Ferdinand de Saussure. Was ihm am ehesten vorschwebt, ist eine "Meta-Linguistik «, wie er es nennt. In diesen Kontext fügt sich auch der gesamte Band Sprechgattungen, in dem durchwegs über Literatur- oder Sprachwissenschaft hinausgreifende Fragen zur Sprache kommen. Bachtin geht es um eine Kommunikologie (die er nicht so nennt), die menschliches Handeln überwiegend als intersubjektives (dialogisches) synchron-diachron differenziertes Sprech-Handeln fasst, auch dort, wo geschwiegen wird.

Gegen die Saussure'sche Dyade/Triade langue - [langage] - parole stehen bei Bachtin »Spracheく (jazyk) als System, >Redeく (reč) als Gesamtheit der Texte, ১Sprechkommunikation`(rečevaja kommunikacija) als gattungsgebundene, wirklichkeitsbezogene Tätigkeit und > ̈̈ußerung`

2 Zur Differenzialdiagnose vgl. die Aufsätze in »Text« 10 (2005), besonders die programmatische Einleitung von Reuß: Text, Entwurf, Werk, S. 1-12. 
(vyskazyvanie) als Artikulation eines werthaltigen Standpunkts.« (S. 224, Anm. 23) Alle Komponenten dieses Systems sind an Handlungen (und damit an das Zwischenmenschliche) gebunden, sodass es nicht überraschen kann, wenn Sprechgattungen immer auch vom Adressatenbezug abhängen. So fasst Bachtin als Einheiten des Sprech-Handelns - und hier richtet sich Bachtins Konzept im Kontext der 1950er Jahre gegen beinahe die gesamte linguistische Forschungstradition - nicht Worte und Sätze (Lexeme und Syntagmen), sondern Äußerungen, die durch Sprecherwechsel begrenzt sind. Ähnlich wie die Bestimmung des Satzes nicht trivial ist und hinsichtlich notwendiger Bestandteile, Funktion oder schlicht dem Umfang (was macht man etwa mit einer Ein-Satz-Erzählung wie Xaver Bayers Wenn die Kinder Steine ins Wasser werfen?3) eine fragwürdige Einheit ist, reicht der Umfang einer Äußerung von einem Einzellaut (etwa einem langgezogenen »Ah!«) bis hin zu einem gesamten Roman (der seinerseits eine Menge dialogischer Äußerungen beinhalten kann), was Bachtins Konzept zunächst kontraintuitiv erscheinen lässt.

Der Titel des einzigen von Bachtin zur Drucklegung vorbereiteten Textes im vorliegenden Band, also Sprechgattungen ${ }^{4}$ bezieht sich auf jene überindividuellen Vereinbarungen, die regeln, wie Äußerungen im Hinblick auf ihre kommunikative Funktion auszusehen haben. Vom Gattungsbegriff der Literaturwissenschaften unterscheidet sich Bachtins "Sprechgattung" in zumindest zweierlei Hinsicht:

Einerseits sind Sprechgattungen der weitere Begriff, indem Bachtin eine Erweiterung vornimmt auf mündliche (etwa: Alltags-)Kommunikation. Sprechgattungen formieren jede mögliche Form sprachlicher Äußerung, sei sie mündlich oder schriftlich verfasst - literarische Gattungen wären also nur ein enger Ausschnitt der allgemeinen Gattungshaftigkeit von Äußerungen. Aufgrund der auf den ersten Blick erkennbaren Offenheit und Unabschließbarkeit der Auswahl an Sprechgattungen kommt Bachtin nicht in die Verlegenheit, eine Taxonomie, Klassifikation oder gar Typologie der Sprechgattungen vorzuschlagen - ein Holzweg, den die literaturwissen-

3 Hier wird `Satz $`$ offensichtlich implizit bestimmt als »das, was durch einen Punkt abgeschlossen ist«, Nebensätze gibt es in Bayers Erzählung erwartungsgemäß zuhauf.

4 Im Original heißt der Text Проблема речевыхжанров (Problema rečevych žanrov, die »Probleme von ... « verschwinden im vorliegenden Band aus dem Titel); an einer Stelle (S. 19) schlägt der vorliegende Band kommentarlos "Redegattungen « als Übersetzung vor. Für die vorliegende Rezension sind an Übersetzungen berücksichtigt worden: Bahtin: Problem govornih žanrova sowie Bakhtin: The Problem of Speech Genres. Zur Frage der Übersetzung u.a. des Titels vgl. Sériot: Généraliser l'unique, bes. S. 40f. 
schaftliche Gattungstheorie bekanntlich seit Jahrhunderten immer wieder einschlägt. ${ }^{5}$

Andererseits nimmt Bachtin über den Grad der Vermitteltheit ${ }^{6}$ eine wesentliche Differenzierung vor:

Besondere Bedeutung hat die grundsätzliche Unterscheidung zwischen primären (einfachen) und sekundären (komplexen) Sprechgattungen (es handelt sich nicht um eine funktionale Unterscheidung). Die sekundären (komplexen) Sprechgattungen - Romane, Dramen, wissenschaftliche Untersuchungen aller Art, die großen Gattungen der Publizistik usw. - entstehen unter Bedingungen einer komplexen, relativ hochentwickelten und organisierten (vor allem schriftlichen) kulturellen Kommunikation - in den Bereichen der Kunst, der Wissenschaft, der Gesellschaftspolitik u.ä. Im Laufe ihres Entstehungsprozesses nehmen sie verschiedene primäre (einfache) Gattungen der unmittelbaren sprachlichen Kommunikation in sich auf und verarbeiten diese. Wo diese Primärgattungen in die Konstitution der komplexen Gattungen eingehen, unterliegen sie einer bestimmten Transformation: Sie verlieren ihren unmittelbaren Bezug zur Wirklichkeit und zu realen fremden Äußerungen; so bewahren etwa Repliken des Alltagsdialogs oder Briefe im Roman ihre Form und Alltagsbedeutung nur auf der Inhaltsebene des Romans und beziehen sich dagegen auf die Wirklichkeit nur über die Vermittlung durch das Romanganze, d.h. als literarisch-künstlerisches, nicht aber als Alltagsereignis. Der Roman als Ganzes ist eine Äußerung gleicher Art wie die Repliken des Alltagsdialogs oder ein persönlicher Brief, aber er ist im Unterschied zu ihnen eine sekundäre (komplexe) Äußerung. / Der Unterschied zwischen den primären und den sekundären (ideologischen) Gattungen ist von größter Bedeutung. (S. 9f.)

Allein dieser Absatz trägt das Potenzial in sich, die Gattungstheorie nachdrücklich zu verschieben. Die Trennung von primären und sekundären Gattungen (oder jedenfalls nach einer vertikal gedachten Schichtung) lässt, so sie konsequent angewendet wird, bei der Diskussion gattungsbezogener Probleme im Bezug etwa auf den Roman vieles deutlicher hervortreten, als das ohne diese Differenzierung möglich wäre. Als Beispiel sei auf den Briefroman verwiesen - Briefe werden von Bachtin bereits in den Vorarbeiten als naheliegendes Exempel zitiert (Dialog I, S. 67; Dialog II, S. 92; Vorbereitende Materialien S. 133 u.ö., davon wird allerdings in den Sprechgattungen verallgemeinernd abstrahiert): Hier ergibt sich eine substanzielle Differenz zwischen dem Brief als Primärgattung und der komplexen Überformung und Primärgattungszitation in einer sekundären Gattung. Vermeiden ließe sich durch konsequentes Auseinanderhalten der beiden Gattungsebenen der Fehler mancher literaturwissenschaftlicher Forschungsliteratur, reale Korrespondenzen und fiktionale Briefromankorrespondenzen implizit

5 Zum Problem der Gattungsklassifizierung vgl. Michler: Kulturen der Gattung, S. 21-27 u.ö.

6 Hier lässt sich, da Kommentar und Nachwort dazu schweigen, nur vermuten, dass dieses Unterscheidungskriterium Bachtins nicht zuletzt auch dem in einer Pendel- oder Gegenbewegung hervorgehobenen Status der Hoch-/Literatur-/Buchsprache geschuldet ist, aus der auch die meisten von Bachtins Beispielen stammen. 
oder explizit für gattungs- oder strukturanalog zu erklären. Ein Brief kann zwar selbst Merkmale einer Sekundärgattung annehmen, wo er z.B. andere Gattungen in sich einschließt, auch kann eine sekundäre Äußerung wie der komplexe Briefroman Werther seinerseits in einer weiteren Metaisierungsoperation nochmals Bestandteil sekundärer (tertiärer) Gattungsaktualisierung werden - doch für beide Fälle liefert das von Bachtin vorgeschlagene Differenzkriterium große Vorteile.?

Doch das ist nur ein Schlaglicht unter vielen möglichen Ansatzpunkten für neue Forschung in- und außerhalb des gattungstheoretischen Rahmens (der sich bei Bachtin, das geht aus den abgedruckten Vorarbeiten hervor, auch aus der zeitgenössischen Diskussion zum Stil entwickelt, vgl. S. 71); ${ }^{8}$ auch für die Bachtin-Philologie im engeren Sinn wie im weiteren einer Geschichte der Philologie und Linguistik in der UdSSR unter Stalin liefert der Band Erkenntnisse: Wissenschaftliches Wirken lässt sich generell nicht im luftleeren Raum erledigen, sondern es trägt (um an Bachtins Terminologie zu bleiben) selbst dialogische Spuren des Kampfes, auch des Autors mit sich selbst anlässlich der beigegebenen Skizzen und Notate. Die Anmerkungen im Apparat beziehen sich etwa zur Hälfte auf solche situativen Kontexte, in denen Bachtins Formulierungen auf Beiträge zur v.a. linguistischen Diskussion antworten; meist sind es ablehnende oder kritische Bezugnahmen, mitunter obligate Zitate aus der Nomenklatura bis zu Stalin, die in den Anmerkungen hervorgehoben werden. Mithilfe des umfangreichen Apparats gelingt es dem Buch, die zeitgenössische Diskussion um Kategorien wie den Stil nachvollziehbar zu machen und Bachtins Konzepte nicht isoliert zu präsentieren, sondern auch in ihrer wahrscheinlichen Entstehungsmotivation zu verorten. Allerdings stellt sich hier die Frage nach der Zielgruppe des Bandes: Wer in die zeitgenössischen Verstrickungen russischer Sprachund Literaturwissenschaften einzusteigen fähig und willens ist, hätte die Übersetzung ins Deutsche, mit all den terminologischen Fallstricken, wohl kaum gebraucht.

7 So ließe sich teilweise die berechtigte Kritik entschärfen, die Definition der primären Sprechgattungen bliebe zu diffus, bei Dusini: Tagebuch, S. 28f., Anm. 35. Zielrichtung Bachtins ist, das geht aus Dialog II hervor, auch die primären Gattungen aus den sekundären heraus zu erforschen, insofern ergänzen die Vorarbeiten Bachtins den veröffentlichten Aufsatz sinnvoll. Die Arbeit von Dusini enthält m.W. eine der frühesten Berücksichtigungen von Bachtins Speech Genres-Aufsatz in der deutschsprachigen Theoriebildung und geht auf dessen Weiterungen im Kontext von Gattungs- und Sprechakttheorie ein.

8 Vgl. auch S. 75: „Der Stil ändert sich, wenn er aus der einen Gattung in eine andere wandert, diese überträgt auf sie die Besonderheiten der vorherigen Gattung. Das Eindringen der Sprechstile in die Literatur dialogisiert die literarischen Gattungen.« Für den Briefroman gilt das jedenfalls. 
Auf der anderen Seite ist gerade die dichte Verwobenheit von allem mit jedem ein Darstellungsproblem, das an die Grenzen der üblichen Buchnavigation führt. So beziehen sich mehr als einmal die Anmerkungen zu einem Text auf drei bis fünf andere Anmerkungen zu anderen Texten und deren Belegstellen, sodass gewissenhaftes Lesen an mehreren Stellen im Band schon rein buch- und blättermechanisch erschwert ist.

Durch die mehrschichtige Anlage der Anmerkungen, die teilweise die Kommentare von Ljudmila A. Gogotišvili, der Herausgeberin der russischen Ausgabe, übersetzend zu übernehmen scheinen, diese aber von Fall zu Fall erweitert und ergänzt, sie aber in einigen Fällen auch nur im Konjunktiv wiedergibt, um Distanz zu den Anmerkungen der russischen Anmerkungsschicht zu signalisieren, entsteht ein Netz von Bezügen, das schwer zu entwirren ist. Eine teilbefriedigende Antwort auf die essenzielle Frage, wer im palimpsesthaften Apparat eigentlich spricht, findet sich erst am Ende der ersten Anmerkung zu den Archiv-Niederschriften (S. 243) und damit an einer Stelle, an der der gewissenhafte Leser bereits 33 Seiten petit gedruckter Anmerkungen hinter sich hat; besonders irritierend ist das Nichtwissen darum, wer spricht, in einem Band, der dieser Frage zentrale Bedeutung einräumt! Unverzeihlich wird dies dort, wo der Apparat die Fußnoten Bachtins stillschweigend in die eigene fortlaufende Zählung übernimmt. Für jene, die das Buch ohne russisches Original oder editorisch saubere Übersetzung vor sich haben: Aus Bachtins Feder stammen, ohne dass dies im Band irgendwie markiert wäre, die Anmerkungen 16, 20, 24, $31,33,35,44,48,59,62,70,75,82,83$ sowie 86 . Einen solchen editionstechnischen Super-GAU hat die englische Übersetzung technisch vor über 30 Jahren durch zwei Apparate zu vermeiden gewusst.

Im Detail des komplexen Buches, in dem ziemlich viel mit ziemlich vielem zusammenhängt und dem deshalb auch manches nachzusehen ist, sind neben solchen unverzeihlichen editorischen Fehlentscheidungen lässliche, aber ärgerliche Fehler entstanden, von denen wenige exemplarisch aufgeführt seien: Von inkonsistenter ss/ß-Schreibung, Tippfehlern wie »Widergabe« (S. 244, Anm. 3) oder »unterstrichten« (S. 306, Anm. 42) und Übersetzungsfehlleistungen (S. 90 die »in der Straße heimischen « Spielarten des Alltags-Dialogs - in der Straße ist höchstens ein Maulwurf heimisch) bis zu nicht durchgeführten satztechnischen Hilfestellungen für den Leser (S. 32, S. 108: funktionsloser Asterisk, S. 277, Anm. 174: Hier und im Weiteren werden solche >durchgekreuzten $`$ Absätze mit einem Strich an der linken Seite und durch kleinere Schrift markiert «, wovon im weiteren Text keine Spur aufzufinden ist), nicht aufgelösten Verweisen (S. 266, Anm. 113: »im oben zitierten Aufsatz«), verzählten Fußnoten (S. 66: zweimal 
Anm. 27) reicht hier das Spektrum, auch willkürlich hinzugefügte größere Absatzabstände (umgekehrt das Wegfallen von Absatzmarkierungen wie in S. 227, Anm. 33) fallen im Abgleich mit der russischen Quelle ins Auge. Mit einem Personenregister und einem Glossar wären dem Leser zusätzliche Navigationsinstrumente an die Hand gegeben gewesen.

Das Nachwort gibt zusätzlich zu Bachtins Überlegungen auch alternative Erklärungsmodelle, die teils auch spätere Reaktionen wie die Bedeutung Bachtins für die Wirkungsästhetik (S. 188) oder die Sprechakttheorie mit einbeziehen - was für Anachronismen sorgt, die man ihrerseits (mit teils beträchtlichem Aufwand, so man nicht zumindest Lebensdaten genannter Personen und Erscheinungsdaten wichtiger genannter Werke im Kopf hat) beim Lesen mit entschlüsseln muss. Im Nachwort, dem solcherart die Erdung an den Text, aber auch an seine Entstehungsbedingungen fehlt, führen die Herausgeberinnen einige Fäden zusammen (andere kommen erst recht disparater zueinander zu stehen). Allerdings bleibt der Leser etwas ratlos zurück, da der Schluss vieles wieder abschwächt. Das überrascht letztlich angesichts der summarisch doch zu würdigenden Editions- und Übersetzungsleistung des vorliegenden Bandes.

Fazit: Ein Grundlagentext des späten Bachtin wird zu Recht wieder entdeckt und liegt nun endlich in einer über weite Strecken kohärenten Übersetzung vor. Dieser Text und das darauf bezogene Materialkonvolut sind theoretisch anschlussfähig unter anderem an die Sprechakttheorie (Austin/ Searle u.a., bei denen Gattung, und damit auch der historische Echoraum jeder Äußerung wider Erwarten keine Rolle spielt!), aber auch an die Literatursoziologie Bourdieus, und daher hochrelevant. Der Verlag hat allerdings als Filterinstitution versagt, indem er ein so offensichtlich unfertiges und fehlerhaftes Buch zum Druck befördert hat. Die in dem Band versammelten Texte werden dennoch für Gattungsfragen noch und wieder hochrelevant sein, das vorliegende Buch vergibt trotz seiner ästhetisch-buchgestalterisch hervorragenden Ausstattung leider durch sowohl editorische als auch editionstechnische Fehlleistungen, durch die Wirrungen der vielschichtigen Zusätze und v.a. des Apparats zahlreiche Chancen; ${ }^{9}$ " hier kann jeweils nur von einem gewissen Abschließungsminimum gesprochen werden« (S. 29).

9 Auf allfällige weitere Übersetzungen von Bachtins Sprech- bzw. Redegattungen ins Deutsche darf gespannt gewartet werden. Im Vorhinein Dank an Kira Elisabeth Kaufmann für sprach- und sachkundige Hinweise. 


\section{Literaturverzeichnis}

Bahtin, Mihail: Problem govornih žanrova. In: Estetika jezičkog stvaralaštva. Hg. S. S. Averincev und S. G. Bočarov; übers. v. Mirjana Grbić. Sremski Karlovci / Novi Sad: Izdavačka knjižarnica Zorana Stojanovića 2013, S. 149-198.

Bakhtin, Michail: The Problem of Speech Genres. Übersetzt v. V. W. McGee. In: Speech Genres and other Late Essays. Hgg. Caryl Emerson, Michael Holquist. Austin: University of Texas Press, S. 60-102.

Bayer, Xaver: Wenn die Kinder Steine ins Wasser werfen. Salzburg: Jung und Jung 2011.

Dusini, Arno: Tagebuch. Möglichkeiten einer Gattung. München: Fink 2005.

Michler, Werner: Kulturen der Gattung. Poetik im Kontext 1750-1950. Göttingen: Wallstein 2015.

Reuß, Roland: Text, Entwurf, Werk. »Text« 10 (2005), S. 1-12.

Sériot, Patrick: Généraliser l'unique: genres, types et sphères chez Bakhtine. »Linx« 56 (2007), S. 37-53. 